\title{
Clinical and immunological features of bronchial asthma with paecilomycosis
}

\begin{abstract}
Background: Infections by the fungi of Paecilomyces genus (PI) were identified among the patients with bronchial asthma (BA), both the carriers of the fungal infection and persons with its acute clinical manifestations.
\end{abstract}

Objective: Our aim was to study the clinical and immunological features of asthma of the Paecilomycosis

Methods: This study was carried out using cases of 143 patients with blood infection by tissue forms of Paecilomyces. A comprehensive survey included: collecting the history of allergy, physical examination, clinical, laboratory and mycological tests. The presence of PI was determined using methods of rapid paecilomycosis diagnosis, serological and cultural studies. Total and specific IgE- and IgG -antibodies to the allergen were identified using the diagnostic kit.

Results: Based on the data of examination and dynamical observation of 143 clinica patients studied the clinical characteristics of atopic and non-atopic forms of asthma. Among them, in 120 (83.9\%), activation of PI was detected: the content of mature spherules of the fungus of the genus Paecilomyces in blood ranged from 8000 to 55000 in $1 / \mu \mathrm{L}$ at a rate of $1000-6000$ in $1 / \mu \mathrm{L}$ (an average of $3417 \pm 433$ in $1 / \mu 1$ ). The development of asthma in them was induced by activation of PI in the blood on the background of acute respiratory viral infection in $74(61.7 \%)$ patients, seasonal and age-related fluctuations of the immune system in $34(28.3 \%)$ patients, stressful effects in $12(10 \%)$ patients. Clinical characteristics of both atopic and non-atopic forms of BA with paecilomycosis were studied. In the asthmatic patients, a resistance to the Paecilomyces fungal infections was mediated by the close connection of cellular and humoral immunity. Under antifungal treatment, pathogens' antigenic effect was reduced, which contributed to the restoration of immunity and the effective control of the number of fungal cells in blood. Immunity is unsterile in the presence of Paecilomyces fungal infections.

Conclusion: Clinical bronchial asthma in paecilomycosis depends on the features of the genetically determined ability of the individual to resist infection with the predominance of a non-atopic variant of the immune response. The resistance to infection by the fungus of the genus Paecilomyces is determined by the close relationship between the cellular and humoral units of immunity.

Keywords: asthma, paecilomyces variotii, dimorphic fungi, paecilomycosis, immunity, adcc
Volume 3 Issue 7 - 2017

\author{
Akhunov Vasiliy Mikhailovich,', Sizova \\ Zhanna Mikhailovna,' Akhunova Alima \\ Muratovna, ${ }^{2}$ Lavrentyeva Tatyana Petrovna, ${ }^{3}$ \\ Aizina Nataliya Liberovna ${ }^{3}$ \\ 'Sechenov First Moscow State Medical University of the Russian \\ Ministry of Health, Russia \\ ${ }^{2}$ Department of Health of the city of Moscow of the Ministry of \\ Health of the Russian Federation, Russia \\ ${ }^{3}$ Department of Health of the city of Moscow, Russia
}

\begin{abstract}
Correspondence: Akhunov Vasily Mikhailovich, Sechenov First Moscow State Medical University of the Russian Ministry of Health, Institute of Vocational Education, Chair of Medical and Social Expertise and Outpatient Therapy, I 17556 Moscow, Fruktovaya str. I2, Russia, Tel +79647965988 , Fax +7499317650I, Email vaahunov@mosmedzdrav.ru
\end{abstract}

Received: November 29, 2017 | Published: December 18 2017
Abbreviations: PI, paecilomyces infections; BA, bronchial asthma; ADCC, antibody dependent cell mediated cytotoxicity; GINA, global initiative for asthma; URTI, upper respiratory tract infection; NSAID, nonsteroidal anti-inflammatory drug; NK, natural killer cell

\section{Introduction}

So far, the question of the etiology and pathogenesis of the intrinsic (non-atopic) form of asthma (one of the most common diseases) remains open. For many years, the prospects for the diagnosis and treatment of intrinsic asthma were associated with the discovery of new facts that will reveal the pathogenesis of the disease. ${ }^{1}$ The latter requires clarification and includes the same picture of the morphological changes in the lungs, as well as the absence of clear immune pathological distinctions in different clinical characteristics of atopic and intrinsic forms of asthma. In bronchial biopsies of patients with intrinsic asthma the same profile of Th-2 helper cytokines and inflammatory cells is detected, as in patients with atopic asthma. ${ }^{2}$
Acute respiratory diseases are the most common factor of aggravation of intrinsic asthma1. In recent years, information about a new form of systemic mycosis caused by Paecilomyces infection appeared. Polymorphism of clinical manifestations of paecilomycosis from nail infections to septic endocarditis was described. ${ }^{3,4}$

Fungi of the genus Paecilomyces are activators of innate chronic persistent infections of the blood, they have dimorphic and opportunistic properties. In nature, the main reservoir of PI is the soil, and in the human body it's the blood. The main route of PI transmission is transplacental. ${ }^{5-7}$ Data of appointments at an allergist office of a Moscow municipal clinic, revealed blood infection caused by tissue forms of the Paecilomyces fungi, either in a form of a carrier or in the form of PI activation with clinical manifestations of allergic disease and/or asthma. ${ }^{8}$ The purpose of the research is to study the clinical and allergic characteristics of asthma of the Paecilomyces etiology and develop new approaches to its laboratory diagnostics available in primary health care. 


\section{Materials and methods}

Studies were conducted in accordance with Helsinki Declaration 2013. A total of 143 ambulatory patients with asthma were studied (who were being observed and treated at the allergist office of a Moscow municipal clinic; men - 51 (41.3\%), women-84 (58.7\%)). Informed consent in written form was obtained from all the participants of study. Most of the patients were between the ages of 41 to 70 years $-88(65 \%)$, the majority of who were women of an older age group $(69 \%)$. The duration of the disease that was estimated form the time of the diagnosis ranged from 7 days to 5years. The diagnosis and the degree of asthma severity were determined in accordance with the GINA criteria.

A comprehensive survey of these patients included: collecting the history of their allergy, physical examination, clinical, laboratory, and biochemical, microbiological, mycological and functional methods of diagnostics. The presence of PI was determined using methods of rapid paecilomycosis diagnosis (quantity of mature spherules and yeast-like cells in $1 \mu 1$ of blood). ${ }^{9}$ As well as serological and cultural studies. To identify cultures by morphological features, were used determinants, ${ }^{10,11}$ as well as articles containing new information about fungi of the genus Paecilomyces. ${ }^{12,19}$ A kit for the diagnosis of allergic diseases and the establishment of the spectrum of significant allergens (manufactured by the "Dr. Fooke laboratorien GmbH", Germany) was used to determine the total IgE and specific IgE-antibodies to environmental allergens and the Paecilomyces fungus, as well as total IgG and specific IgG-antibodies to the Paecilomyces fungus allergen. The results of the study were processed by the method of summary statistics using the t-test (Student t-test). All results of the study are presented as the arithmetic mean of the sample and the standard deviation $(\mathrm{M} \pm \mathrm{m})$; differences were considered reliable at a significance level of $\mathrm{p}<0.05$.

\section{Results}

The data of appointments at the allergist office of a Moscow municipal clinic indicated infection of the blood by caused by tissue forms of Paecilomyces fungus in all patients with asthma. In accordance with the results of express - diagnostics of paecilomycosis as well as serological and cultural studies all patients with asthma were divided into 2 groups. The first group consisted of 23 (16.1\%) patients with atopic asthma, tissue forms of Paecilomyces fungus were detected in their blood with no signs of activation. These patients suffered from asthma attacks after contact with environmental allergens. All patients were aged between 16 and 29years (average

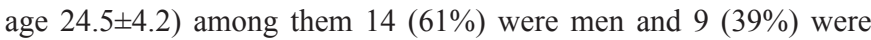
women. The duration of asthma in these patients ranged from 1year to 5 years (average $3.07 \pm 1.97$ years). All patients had elevated total $\operatorname{IgE}$ and specific IgE antibodies to environmental allergens. According to their allergic histories, 11 (47.8\%) patients had relatives who suffered from allergic diseases. Increased sensitivity to house dust in the form of nasal congestion, sneezing, itching eyes, watery eyes and asthma attacks was noted in $13 \%$ of patients. Contact with pets (cat, dog, hamster, etc.) provoked symptoms of allergy and asthma in $17.4 \%$ of patients; $35 \%$ of the patients mentioned the suffering from seasonal rhinitis, conjunctivitis and asthma attacks associated with flowering trees and grass; $34.6 \%$ of the patients had increased sensitivity to various types of environmental allergens. In addition, there were indications of skin rashes and itching caused by consumption of nuts, eggs, and cow's milk; in patients with allergies to birch pollen this was caused by the consumption of apples, carrots, cherries and vitamins of group B. The quantitative content of mature fungal spherules and yeast-like Paecilomyces fungus cells ranged from 1000 to 6000 in 1/ $\mathrm{ml}$ in the blood of the patients from this group, and was comparable to the control group indicators ( $>0.05$ ), which allocated them to the group of Paecilomyces carriers (Table 1).

The second group consisted of $120(83.9 \%)$ patients with an average age of $51 \pm 7.3$ years suffering from asthma and symptoms of PI activation in the blood. In $74(61.1 \%)$ patients asthma attacks occurred for the first time or became 2-3 times more frequent weeks after an onset of URTI, which progressed with an increase in body temperature to $38-40^{\circ} \mathrm{C}$ for $1-3$ days, chills, malaise, congestion of the nasal passages and release of watery secretions. Rare coughs in the beginning of the disease gave way to a paroxysmal expiratory type dyspnea after 7-10days. Asthma attack occurred predominantly at night or early in the morning. In $34(28.3 \%)$ patients with the active PI in the blood, asthma attacks first emerged and subsequently exacerbated in early spring and late autumn. $12(10 \%)$ patients in this group pointed out a connection between the disease and stress exposure.

$18(15 \%)$ of the patients had relatives with a history of allergic diseases, $15(12.5 \%)$ patients had allergic reaction to penicillin antibiotics, aspirin or NSAIDs. In $5(7.46 \%)$ patients, with PI activated by URTIs, the administration of penicillin type drugs caused systemic reactions in the form of a generalized rash and asthma attacks. 35 $(29.16 \%)$ patients with the active PI in the blood displayed increased sensitivity to dust of moldy wood and old paper in the form of nasal congestion, sneezing, itchy eyes, watery eyes, itching of the skin and asthma attacks. Food allergies were noted in $10 \%$ of patients, with red foods (red fish) as the dominate allergens. The duration of the disease ranged from 7 days to 12 weeks with an average of $-4.1 \pm 1.2$ weeks. The average content of mature of Paecilomyces fungus spherules in the blood for the group was $17500 \pm 755$ in $1 / \mu 1$. Blood cultures of $42(35 \%)$ patients on solid nutrient media showed growth of Paecilomyces fungus with the prevailing of Paecilomyces variotii Bainier (1907) (58\%) fungus. In 49 (40.8\%) patients with activate PI asthma was mild persistent, in $71(59.2 \%)$ patients it was moderate. In the blood of patients with mild asthma the quantitative content of mature Paecilomyces fungus spherules ranged from 8000 to 17000 in $1 / \mu 1$ with an average of $14020 \pm 416$ in $1 / \mu 1$ and a norm of $3417 \pm 433$ in $1 / \mu 1(\mathrm{p}<0.001)$

In the hemograms of these patients, small eosinophilia was noted from 5 to $7 \%$ with an average of $5.2+0.48 \%$, and / or lymphocytosis $38.5+1.0 \%$ (Table 1). Clinical symptoms of the disease in patients with mild asthma of Paecilomyces etiology have been studied in $71(59.2 \%)$ patients - 28 men (39\%) and 43 women (61\%). The quantitative content of mature spherules of Paecilomyces fungus in the blood of patients with asthma of moderate severity ranged from 16000 to 55000 in $1 / \mu 1$, an average of $17560 \pm 326$ in $1 / \mu 1$. The hemogram revealed severe blood eosinophilia- $14.4 \pm 0.58 \%$, lymphocytosis and monocytosis.

Of the 120 patients with asthma and active Paecilomyces infection in the blood total IgE was normal or reduced in 67 (55.8\%) patients. Specific IgE- antibodies to environmental allergens were detected in the blood of $10(14.92 \%)$ patients. $7(8.4 \%)$ patients had no IgE hyper production with no connection to atopy, and $46(38.33 \%)$ patients with asthma and active PI in blood, had elevated total IgE and specific IgEantibodies to environmental allergens. $29.16 \%$ of them were suffering 
from seasonal rhinitis, conjunctivitis and/or asthma attacks caused by pollen of plants or herbs. $33.33 \%$ of patients suffered from asthma attacks provoked by contact with pets. Combined increased sensitivity to epidermal, consumer and pollen allergens was identified in $37.51 \%$ of patients. Allergic reactions and/or asthma in patients of this group were observed since childhood and have occurred sporadically when in contact with the allergens.

The nature and severity of asthma changed for 7-10 days after onsets of URTI and influenza: asthma attacks became more frequent, occurred mostly at night or early in the morning, and tended to be more prolonged. Respiratory symptoms along with concomitant diseases of other organs and symptoms of infection and intoxication in the form of general weakness, malaise and sweating were typical for the entire group of patients with activate PI. $60.25 \%$ of patients in this group suffered from concomitant diseases of the cardiovascular system, $15.4 \%$ from arthralgia, and 9.8\% from polyneuropathy. 32 (26.6\%) of the patients with active PI in the blood had cutaneous manifestations in the form of localized or widespread lesions. Among them were cases of erythema, maculopapular rash, urticaria rash with acute circumscribed edema, bullous lesions and psoriatic plaques. Usually, the appearance of skin lesions preceded the development of asthma attacks.

Out of the 26 patients with atopic immune responses 15 (57.69\%) patients had allergen specific IgE- antibodies to the Paecilomyces fungus, $21(80.7 \%)$ patients had allergen specific $\operatorname{IgG}$ - antibodies, the combination of specific IgE- and IgG - antibodies to the Paecilomyces fungus were found in $14(53.84 \%)$ patients and $7(26.9 \%)$ patients had only specific IgG antibodies (Table 2$)$. In 4 (15.3\%) of these patients with active PI of the blood in an acute activation phase antibodies to the Paecilomyces fungus were not identified. In a group of 20 patients with non-atopic immune responses to the activation of PI in the blood allergen specific IgE-antibodies to the Paecilomyces fungus were identified in $2(10 \%)$ patients, allergen specific IgG antibodies were identified in $11(55 \%)$ patients and their combination was identified in $1(5 \%)$ patient. Allergen specific antibodies were not detected in four patients from this group with the PI of the blood in an acute activation phase. The results of specific allergy research indicate the fundamental role of specific IgG-antibodies in the protection against Paecilomyces fungus infections and a close relationship of cellular and humoral immunity. According to the literature, the development or exacerbation of the course of bronchial asthma in paecilomycosis, was observed in patients with a lowered level of total $\operatorname{IgG} .{ }^{13}$ Low level of $\operatorname{IgG}$ was also noted in the sera of the mothers and their newborns with intrauterine pneumonia initiated by the fungus of the genus Paecilomyces. ${ }^{6}$ The cellular composition of peripheral blood indicated that eosinophils, basophils, lymphocytes and monocytes were being implemented for the realization of phagocytic and cytotoxic functions (Table 1). According to literary sources eosinophils and NKs are deployed for the destruction of target cells too large for phagocytosis with the aid of specific mediating antibodies. ${ }^{14}$ In individuals with atopic immune responses the prevalence of eosinophilic protection mechanism was noted (Figure 1A), in individuals with non-atopic immune responses the lymphocytic defense was dominant (Figure 1B). There are several additional indicators of participation of erythrocytes in fungicidal action, including (a) coverage of erythrocyte's membranes by endospores, (b) erythrocyte's adhesion to large fungal cells, and (c,d) the presence of small fungal cells or elements of fungal cytoskeleton in erythrocyte's cytoplasm (Figure 1C \& 1D).

Table I Cellular composition of peripheral blood in patients suffering from bronchial asthma with different profiles of immune response during infections by the Paecilomyces fungus

\section{S. The immunological profile of no patients with asthma}

The content of mature spherules of the paecilomyces fungus in the blood ( in $1 / \mathrm{ml}$ )

\section{Cellular composition of peripheral blood (\%)}

\begin{tabular}{|c|c|c|c|}
\hline \multirow{3}{*}{1} & \multirow{3}{*}{$\begin{array}{l}\text { Atopic without PI activation. } \\
\text { Paecilomyces carriers }\end{array}$} & \multirow{3}{*}{$3608 \pm 328$} & Eosinophils-3.4 \pm 0.29 \\
\hline & & & Lymphocytes- $26.4 \pm 0.87$ \\
\hline & & & Monocytes-5.8 \pm 0.15 \\
\hline \multirow{4}{*}{2} & \multirow{4}{*}{$\begin{array}{l}\text { Atopic with active PI in the blood. } \\
\text { Paecilomycosis }\end{array}$} & \multirow{4}{*}{$17580 \pm 326 *$} & Eosinophils- $14.4 \pm 0.58 *$ \\
\hline & & & 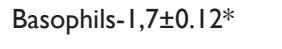 \\
\hline & & & 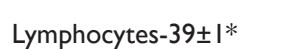 \\
\hline & & & Monocytes.-8 $\pm 0.3 *$ \\
\hline \multirow{5}{*}{3} & \multirow{5}{*}{$\begin{array}{l}\text { Non-atopic, with active PI in the } \\
\text { blood. Paecilomycosis }\end{array}$} & \multirow{5}{*}{$17500 \pm 755^{*}$} & Eosinophils- $5 \pm 0.48$ \\
\hline & & & Basophils-I.6 $\pm 0.99 *$ \\
\hline & & & \\
\hline & & & Lymphocytes-40 $\pm 0.99 *$ \\
\hline & & & Monocytes-I I $\pm 0.5^{*}$ \\
\hline
\end{tabular}


The intensity of positive reactions to environmental allergens in patients with atopic immune responses and active PI in the blood reached 4+-6+ and indicators of positive reactions to Paecilomyces fungus allergen were mostly in the $1+$ and $2+$. The lowered immediate hypersensitivity towards Paecilomyces fungus was apparently due to the generalized spread of the pathogen that activated the immunosuppressive regulatory mechanisms for the suppression of systemic anaphylactic reactions. Including systemic antimycotics in the complex treatment of patients with asthma with the PI of the blood in an acute phase of activation restores immune control over the number of fungal cells, eliminating the development of allergic symptoms and exacerbations of asthma; this allows achieving longterm remission of the disease or clinical recovery. ${ }^{9}$

Table 2 Specific IgE-and IgG-antibodies to the Paecilomyces fungus allergen in the blood of patients with bronchial asthma

\begin{tabular}{|c|c|c|c|c|}
\hline Indicators & $\begin{array}{l}\text { Patients with Atopic Asthma and Active PI } \\
\text { (N 26) }\end{array}$ & $\%$ & $\begin{array}{l}\text { Patient with Non-Atopic Asthma and Active PI } \\
\text { (N 20) }\end{array}$ & $\%$ \\
\hline $\lg E+$ & 15 & 57.69 & 2 & 10 \\
\hline $\operatorname{lgG}+$ & 21 & 80.7 & 11 & 55 \\
\hline $\lg \mathrm{E}+\lg \mathrm{g}+$ & 14 & 53.84 & I & 5 \\
\hline IgE- IgG- & 4 & 15.3 & 4 & 20 \\
\hline
\end{tabular}

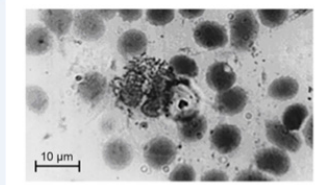

(a)

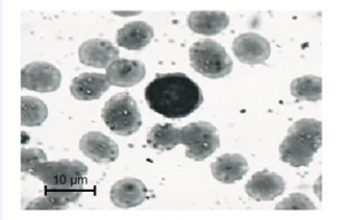

(c)

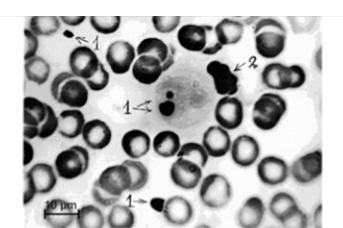

(b)

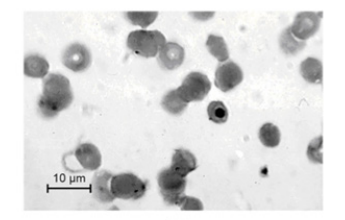

(d)
Figure I Cytological characterization of the cell composition of the peripheral blood of BA patients with Paecilomyces.

a) Smear of peripheral blood of a patient with atopic профиль bronchial asthma with paecilomycosis. Sprawled eosinophil on the surface of mature spherule with concentration of granules around the fungal membrane. Romanowsky-Giemsa stain.

b) Smear of peripheral blood of a patient with non-atopic профиль bronchial asthma with paecilomycosis.. A mononuclear realizing phagocytic and cytotoxic functions: I - clearly visible absorbed endospores in the cytoplasm, 2 - a large yeast-like cell fixed to its surface. Gram-Weigert stain.

c) Peripheral blood patient of bronchial asthma by activation of Paecilomyces infection in the blood. In the view erythrocytes, with fixed in their shells, or located in their cytoplasm endospores of the fungus genus Paecilomyces. Endospores round or yeast-like form. At the center, it is seen mature tissue fungal cell with dual-capsule containing therein endospores. Giemsa - Romanowski stain.

d) Preripherial blood of a BA patient with Paecilomyces fungal infection. Small fungal cells or elements of fungal cytoskeleton are observed in the cytoplasm of some erythrocytes. Romanovsky-Gimza stain.

\section{Discussion}

Paecilomyces fungi are widespread soil microorganisms living in different geographical areas. They often contaminate the livestock feed thus leading to spoilage of food, paper and leather10. Starting from the middle of the 1960 s, they attract the attention of clinicians as a causative agent for septic endocarditis. ${ }^{15}$ Pneumonia, sepsis, anemia, ${ }^{16,17}$ and other types of pathologies provoked by the immune deficiency states. Moreover, they can induce dangerous respiratory tract infections such as allergic alveolitis and BA. ${ }^{18}$ It is known that Paecilomyces variotii Bain. (1907) and Paecilomyces viridis (Chamaeleomyces viridis comb. nov.) fungi are dimorphic. ${ }^{19}$ The tissue parasitic forms are spherules and yeast-like cells.

The results Иf this study demonstrate that patients with atopic BA can be the carriers of PI. The long-term persistence of tissue forms of P. variotii in the blood in the form of carrier may indicate its weak pathogenic properties and suppression of the host's immune response. The immunity is unsterile during Paecilomyces fungus infections. The transition from a carrier of Paecilomyces to symptomatic paecilomycosis occurs under the influence of various factors inhibiting the activity of cellular and humoral immunity, the interaction of which determines the resistance to PI. The development of the pathological process during an uncontrolled multiplication of fungal cells is realized through lipid metabolites of the Paecilomyces fungus and hypersensitivity mechanisms induced by the increased levels of fungal antigen.

The clinical manifestation of paecilomycosis in the form of asthma with the activation PI in the blood, is mediated by a type 1allergic reaction with the participation of specific IgE-antibodies to the Paecilomyces fungus allergen. Thus the specific IgE antibodies play a dual role: cause mast cell degranulation with the release of biologically active substances, including histamine, but also ensure the fixation of eosinophils on the capsules of fungal cell for the realization of ADCC. This process is accompanied by the development of allergic symptoms and asthma attacks. The hyperreactivity of bronchial walls mediates their chronic immune inflammation and of the peribronchial tissue that is aimed at the destruction of fungal cells and the prevention of their spread into the lung tissue. Furthermore, during the formation of the hyperreactivity of the bronchial walls lipid metabolites of parasitic forms of Paecilomyces fungus are involved, particularly exogenous fungal phospholipase A2. ${ }^{20}$ It is known that its effects on cellular phospholipid membranes of microenvironment cells causes the formation of arachidonic acid metabolites which act directly on a variety of target cells including afferent and efferent nerves and cause spasms of smooth muscle. ${ }^{21}$ 
The shift from a carrier of Paecilomyces to symptomatic paecilomycosis with the development of asthma of the paecilomycosis etiology may worsen atopic forms of bronchial asthma, which is often preceded by activation of PI in blood of the Paecilomyces carriers. The pathogenic factors are exoallergens, the Paecilomyces fungi endoallergens, and the chronic immune inflammatory processes in the lung tissue, which lead to the aggravation of BA in these patients. As the disease develops, the asthma attacks from episodic become more frequent and prolonged. Without etiotropic treatment, an acute severe asthma is typically developed. Thus, the characteristic features of BA with PI are the presence of the internal infection, the prevalence of clinical manifestations of a non-atopic BA, and the connection with the acute respiratory infections. These features allow one to classify this disease as a kind of the intrinsic form of asthma. The best results and practical outcomes of the express diagnosis of paecilomycosis can be achieved in combination with specific diagnosis of Paecilomyces fungus infections, which is available in the primary care laboratories. Rapid diagnostics of PI activation in the patient's blood facilitates the timely etiotropic treatment and the achievement of long-term remission or clinical recovery. ${ }^{9}$

\section{Conclusion}

Paecilomyces variotii-causes a new type of pathogen opportunistic mycosis, has dimorphism and hemotropic properties. When disseminated into the lung tissue it causes the development of an inflammatory reaction in the bronchial wall that initiates hyperreactivity and bronchial obstruction with a clinical manifestation of symptoms of bronchial asthma. Depending on the profile of the individual's immune response to infection, various pathogenetic mechanisms of asthma development and the forms of its clinical manifestation are formed: atopic, non-atopic, and their combined variant against the already persistent atopic form in the carrier at reactivation of the paecilomycosis infection in the blood.

\section{Acknowledgements}

None.

\section{Conflict of interest}

The author declares no conflict of interest.

\section{References}

1. http://www.respiratory-thessaly.gr/assets/files/GINAReport2007.pdf

2. Humbert M, Corrigan CJ, Kimmitt P, et al. Relationship between il-4 and il-5 mrna expression and disease severity in atopic asthma. $\mathrm{Am} \mathrm{J}$ Respir Crit Care Med. 1997;156(3):704-708.

3. Aguilar C, Pujol I, Sala J, et al. Antifungal susceptibilities of Paecilomyces species. Antimicrob Agents Chemother. 1998;42(7):1601-1604.

4. Pastor FJ, Guarro J. Clinical manifestations, treatment and outcome of Paecilomyces lilacinus infections. Clin Microbiol Infect. 2006;12(10):948-960
5. Ahunova AM. Peacilomycosis. Probl Tuberc. 1989;66(8):38-42.

6. Abduhalikov-zade GA. The Role of Paecilomyces in the Development of Intrauterine Pneumonia. Samarkand, Uzbekistan; 1997.

7. Akhunova AM, Abduhalikzade GA, Abdusalyamov AA. Data of the epidemiologic study on maternal and neonatal peacilomycosis in the Samarkand region. In: Sergeeva YV, editor. Advances of Medical Mycology. Russia: The National Academy of Mycology; 2007. p. 188189.

8. Akhunov VM, Sizova ZM. The prevalence of asthma of the Paecilomyces etiology and possible pharmacotherapy for outpatients. Biomedicine. 2010;5(3):28-29.

9. Akhunov VM. Features of Bronchial Asthma with Paecilomycosis. Germany: Lambert Academic Publishing; 2014.

10. Samson RA. Paecilomyces and some allied hyphomycetes. Stud Mycol. 1974;6:1-119.

11. Hoog GS, Guarro J, Gene J, et al. Atlas of Clinical Fungi. 2nd ed. Spain: Centraalbureau voor Schimmelcultures. Utrecht/Universitat Rovira i Virgili; 2000. 830 p.

12. Houbraken J, Verweij PE, Rijs AJ, et al. Identification of Paecilomyces variotii in clinical samples and setting. J Clin Microbiol. 2010;48(8):P2754-P2761.

13. Ahunova AM, Shustova VI. Clinical and immunological characteristics of paecilomycosis. Klin Med. 1991;69(3):64-67.

14. Haitov RM. Immunology. Russia: GEOTAR - Media; 2006.

15. Uys CG, Don PA, Schrire V, et al. Endocarditis following cardiac surgery due to the fungus Paecilomyces. South African Med J. 1963;37(51):1276-1280.

16. Dehkan-Khodjaeva NA, Shamsiev SS, Shakirova RY, et al. The role of Paecilomyces in the etiology of prolonged and recurrent bronchopulmonary diseases in children. Pediatr M. 1982;60(9):12-14.

17. Alimdzhanova RY. Clinical variants in the development of fungal diseases caused by fungi of the genus Paecilomyces. Ther Arch Moscow. 1993;65(11):53-55.

18. Akhunova AM. Infectious-allergic bronchopulmonary paecilomycosis. Ter Arkh. 1991;63(10):19-24.

19. Sigler L, Gibas CFC, Kokotovic B, et al. Disseminated mycosis in veiled chameleons (Chamaeleo calyptratus) caused by Chamaeleomyces granulomatis, a new fungus related to Paecilomyces viridis. J Clin Microbiol. 2010;48(9):3182-3192.

20. Yulaev MF, Akhunova A. Some properties of the exogenous phospholipase A2 produced by the mycelium of the fungus Paecilomyces viridis. Probl Med Chem. 1998;45(3):223-226.

21. Gershwin ME. Bronchial Asthma. USA: Grune \& Stratton; 1981. 